\title{
Corela
}

Cognition, représentation, langage

HS-30 | 2020

Phonétique, littérature et enseignement du FLE :

théories et recherches

\section{Phonétique, littérature et enseignement du FLE : théories et recherches}

Introduction

\section{Florentina Fredet et Christos Nikou}

\section{(Q) OpenEdition \\ Journals}

Édition électronique

URL : http://journals.openedition.org/corela/9987

DOI : $10.4000 /$ corela.9987

ISSN : $1638-573 \mathrm{X}$

Éditeur

Cercle linguistique du Centre et de l'Ouest - CerLICO

\section{Référence électronique}

Florentina Fredet et Christos Nikou, « Phonétique, littérature et enseignement du FLE : théories et recherches », Corela [En ligne], HS-30 | 2020, mis en ligne le 13 avril 2020, consulté le 22 mai 2020. URL : http://journals.openedition.org/corela/9987 ; DOI : https://doi.org/10.4000/corela.9987

Ce document a été généré automatiquement le 22 mai 2020

\section{(c) (i) (2)(2)}

Corela - cognition, représentation, langage est mis à disposition selon les termes de la licence Creative Commons Attribution - Pas d'Utilisation Commerciale - Partage dans les Mêmes Conditions 4.0 International. 


\title{
Phonétique, littérature et enseignement du FLE : théories et recherches
}

\author{
Introduction
}

Florentina Fredet et Christos Nikou

1 Pour acquérir une langue vivante il faut, bien évidemment, apprendre à la parler (car toute langue est parlée avant d'être écrite), mais cela n'est possible que si l'on connaît sa prononciation. En effet, chaque langue possède un nombre limité de sons qui sont fixés par la communauté linguistique qui la parle. Cependant, la maîtrise d'une bonne prononciation («compétence phonétique ») suppose, à la fois, un enseignement spécifique et un entraînement systématique qui aideront l'apprenant à (mieux) percevoir et produire les unités sonores de la langue dans des contextes donnés.

2 Jusqu'à l'avènement des méthodes ayant pour objectif de « faire parler » l'apprenant, la primauté accordée à l'écrit dans le processus d'enseignement de la langue française minimise le traitement pédagogique de l'oral. Dépendant souvent d'une source écrite (i.e. le manuel), la pratique de l'oral - et par voie de conséquence celle de la prononciation - a peu de place dans le cours de langue. Cette situation se reflète d'ailleurs dans les difficultés de perception et d'expression des apprenants.

3 Malgré le retour « timide » auquel on assiste ces dernières années de l'enseignement de la phonétique en classe de langue étrangère, les méthodes "générales » de Français Langue Étrangère ${ }^{1}$ (FLE) continuent de conférer peu de place aux activités phonétiques qui, de surcroit, sont soit insuffisantes soit présentées dans un ordre aléatoire sans répondre aux véritables besoins des apprenants ${ }^{2}$ (Dufeu, 2008). À cela pourrait s'ajouter la formation des enseignants :

Le problème principal réside dans la formation des enseignants qui reste, en général, succincte dans ce domaine. Les enseignants disposent de peu de fondements théoriques utiles pour orienter et éclairer leur pratique. D'une manière générale, on enseigne peu les connaissances de base sur les caractéristiques prosodiques (rythme et mélodie) de la langue cible et encore moins sur les moyens de les transmettre. L'aspect pratique se réduit le plus souvent à la présentation de 
corrections confinées dans le domaine des sons et faisant essentiellement appel à la méthode articulatoire. Les études universitaires se limitent trop souvent au domaine de la phonologie (ibid.).

4 Les directives du Cadre européen commun de référence pour les langues ${ }^{3}$ (CECRL) rejoignent les propos d'Élisabeth Guimbretière (1994:5), pour qui la phonétique est « la discipline fondamentale de l'étude de l'oral, notamment dans l'enseignement/apprentissage d'une langue ». Cependant, la phonétique est peu enseignée dans les classes de FLE, surtout à l'étranger, ce dont témoignent les distorsions phonétiques que les spécialistes ont maintes fois mises en évidence : prononciation inappropriée de tel ou tel son par les anglophones, germanophones, arabophones, nipponophones...

5 Cessant d'être une science de pure description pour devenir une science d' «intervention» dans la classe de langue, la phonétique doit être intégrée dans la formation initiale des enseignants de FLE. Par le biais de la correction phonétique, l'enseignant peut intervenir alors efficacement, tout d'abord sur la réception auditive de l'apprenant, en s'assurant que ce dernier a bien « entendu » ce qui a réellement été prononcé et non ce qu'il croit avoir été prononcé car, pour identifier correctement le sens, il doit d'abord identifier correctement le support phonique (d'où la nécessité de développer une nouvelle façon d'écoute de la chaîne parlée) puis, sur sa production sonore, en facilitant concrètement sa prononciation par le travail articulatoire. Dans cette perspective, l'enseignant se doit d'aborder, en même temps que les faits de langue (morpho-syntaxiques, sémantiques, lexicologiques), les faits liés à son oralité.

6 En d'autres termes, cette discipline majeure des sciences du langage contribue à faire apprendre les sons du français et à en améliorer la prononciation et, partant, la production orale, par des activités de sensibilisation/discrimination/perception, d'entraînement articulatoire... C'est en ceci justement qu'elle rejoint la littérature : la prose est faite pour être lue, la poésie pour être récitée ${ }^{4}$, le théâtre pour être joué, le conte pour être représenté/raconté aux enfants, la chanson pour être chantée, le matériau littéraire étant, au moins, autant sonore que graphique. La poésie et le théâtre et, dans une moindre mesure, le roman, relèvent des poétiques phoniques ${ }^{5}$, selon l'expression d'Anne Übersfeld (1997), car liés à la déclamation par leur rapport avec l'oralité et le dire. Ces arts de la parole par excellence favorisent la matérialisation des potentialités du système phonémique et prosodique.

7 Par ailleurs, certains types de textes (poésies, chansons, pièces de théâtre, comptines, slogans publicitaires, slams, discours politiques...) visant une harmonie sonore censée retenir l'attention de l'auditeur, contiennent des éléments phonétiques importants qui, pour être compris/appréciés impliquent un minimum de connaissances phonétiques. Ces textes littéraires ou à caractère littéraire, dont les particularités phonétiques (i.e. figures de style) occupent une place très importante, visent, de par leur richesse culturelle, leur dimension esthétique et leur force émotionnelle/évocatrice, à susciter l'intérêt des apprenants, favorisent la communication orale, et facilitent la mémorisation d'une prononciation et d'une intonation conformes à la langue cible. Mais, tout texte peut-il être littéraire? Comment peut-on identifier l'objet littéraire, selon le terme de Gérard Genette?

L'objet littéraire n'existe que par lui ; en revanche il ne dépend que de lui, et, selon les circonstances, n'importe quel texte peut être ou n'être pas littérature, selon qu'il est reçu (plutôt) comme spectacle ou (plutôt) comme message: l'histoire littéraire est faite de ces aller-retour et de ces fluctuations. C'est dire qu'il n'y a pas à proprement parler d'objet littéraire, mais seulement une fonction littéraire qui 
peut investir ou délaisser tour à tour n'importe quel objet d'écriture (Genette, 1966 : 146-147). concerner tous les niveaux d'apprentissage et tous les âges (enfants, adolescents, adultes), selon le texte littéraire et le genre choisis car «la littérature est faite de langage... [que] les écrivains [...] font vivre [...] célèbrent, aiguisent, transforment, parce que le langage est vivant par eux, à travers eux » (Le Clézio, 2008). Qu'il s'agisse de répondre à des besoins linguistiques, culturels, intellectuels et/ou esthétiques, les textes littéraires constituent des supports d'enseignement/apprentissage à part entière, parfaitement exploitables en cours de FLE. Mais pour les aborder dans toutes leurs dimensions (en lecture silencieuse ou à haute voix) et en apprécier le contenu, il faut savoir les «réduire» en leurs composantes : sons, groupes de mots, groupes de sens.

Si l'on veut que l'apprenant étranger véhicule « du sens » dans ses communications en langue cible, il doit avant tout être capable de produire des suites phoniques conformes, ce qui implique la maitrise de la structuration syntaxico-intonative de l'énoncé et des traits spécifiques du phonétisme français. Un énoncé (quel qu'il soit), en dehors du sens qu'il véhicule, reste également une substance phonique, et ce, autant dans le cas d'un énoncé oral que dans un écrit. Ce dernier nous confronte à une graphie, d'abord, mais le lecteur, dans sa langue maternelle, « entend » en même temps qu'il lit : la graphie entraîne immédiatement la phonie en une sorte de voix intérieure. Il est donc nécessaire que l'apprenant, qui lit/entend avec sa propre prononciation (plus ou moins juste), s'habitue à «placer » sa phrase dans un mouvement phonatoire d'ensemble comportant simultanément : la prononciation des sons, la distribution des syllabes accentuées (pour le rythme), les phénomènes d'enchaînement et de liaison, le maintien du timbre vocalique (phénomène de stabilité), l'intonation (la mélodie) de l'énoncé pour favoriser la compréhension dans la communication parlée, rapprocher sa production autant que possible de celle d'un natif... À cet effet, les textes littéraires constituent un support intéressant pour exercer l'apprenant étranger à dépasser les difficultés ${ }^{6}$ de prononciation (et/ou à s'exprimer devant ses pairs) et à appréhender la civilisation et la culture françaises en même temps. Le scripteur (poète, romancier, dramaturge...) compte sur la compétence artistique du lecteur/auditeur pour retrouver la symbolisation sonore de l'écrit, il est donc indispensable que nos apprenants puissent comprendre, apprécier ce qu'ils lisent/entendent. Le poète, par exemple, est avant tout celui qui connait, de science profonde, les ressources du langage, «les accords du son et du sens ", « les effets psychiques que produisent les groupements de mots et physionomies de mots »; il a recours aux "audaces » les plus inattendues et dissout le sens des mots qu'il emploie dans un bain de sonorités harmonieuses. Il en va 
de même pour le romancier, l'écrivain, le dramaturge, le parolier, le concepteur de slogans publicitaires ${ }^{7}$, le compositeur...

11 Si les recherches concernant les difficultés de prononciation spécifiques aux différents groupes linguistiques (ce qui relève de la phonétique contrastive) ne manquent pas, rares sont celles axées sur les apports de la littérature à l'enseignement de la phonétique $^{8}$. Les textes littéraires ${ }^{9}$ (tous genres confondus), issus d'un véritable travail sur la langue ${ }^{10}$ (par des procédés phonématiques - i.e. structuration sonore, prosodique, rythmique - et sémantiques), peuvent très avantageusement venir en complément d'un cours de phonétique selon les besoins des apprenants et les objectifs préalablement fixés, contribuant ainsi au perfectionnement de la perception et de la production. Cependant, le choix judicieux des textes littéraires en fonction des sons/phénomènes phonétiques qu'ils contiennent constitue une tâche difficile pour l'enseignant n'ayant pas de formation spécifique en phonétique.

Pour dédramatiser l'apprentissage de la phonétique, - discipline à la réputation aride, redoutée par les enseignants et dont la place et l'importance ont connu une grande variabilité au cours des âges - il convient de s'interroger sur ses raisons d'être et sur son rôle dans la communication ${ }^{11}$ (Sauvage et Billières, 2019). Ainsi, pour améliorer les capacités de compréhension, d'interaction et de production orales de ses apprenants, l'enseignant devra posséder un minimum de bases théoriques et méthodologiques pour préparer et animer régulièrement des activités de pratique/correction phonétique, afin qu'enseigner/apprendre la langue étrangère devienne un plaisir.

13 Le recueil d'études ici réunies s'adresse aux chercheurs en langue et littérature françaises, en didactique de la littérature, en stylistique ou, plus généralement, en sciences du langage, mais aussi, et surtout, aux enseignants de FLE qui abordent également la littérature dans leurs pratiques de classe (notons que des rubriques « culture » qui proposent, entre autres, des extraits littéraires, apparaissent de plus en plus souvent dans les méthodes de FLE), ainsi qu'à ceux qui, faute d'une formation spécifique et donc d'outils et de pratiques d'enseignement et de correction appropriées, se trouvent démunis faces aux difficultés (qu'ils arrivent cependant à identifier) de leurs apprenants.

14 C'est dans les directions et la problématique esquissées ci-dessus que se sont effectivement déployées les différentes contributions :

15 Le premier axe, "Aspects théoriques et cadrages didactiques", met en valeur l'utilisation du texte littéraire dans l'enseignement/apprentissage de la phonétique en FLE. Cet axe est organisé de manière à ce que soient précisés les apports théoriques dans la pratique de l'intégration conjointe de la prononciation et de la littérature, sans oublier les rapports entre oralité et écrit. Ces éclairages théoriques nous permettent de faire le point sur les pratiques, techniques et outils, et d'établir des critères et angles d'attaque qui, d'après les avancées réalisées en phonétique, révèlent les besoins et les objectifs déjà fixés ici ou là, selon l'âge et le niveau des apprenants, ou à fixer, selon les démarches diagnostiques de l'enseignant; ces critères, dont l'enseignant doit absolument tenir compte, peuvent nous donner des pistes pour le choix des textes littéraires exploitables en cours de phonétique. Dans son article d'ouverture intitulé "Identifier les contenus phonétiques d'un texte littéraire», Bertrand Lauret se propose de construire les bases théoriques d'une exploitation phonétique du texte littéraire. En faisant le point sur les relations, tout aussi délicates que complexes, entre l'oral et l'écrit en didactique du FLE, il avance quelques propositions d'exploitation phonétique 
du texte littérature tout en procédant à une identification des contenus phonétiques dont l'objectif principal est d'aider l'apprenant et/ou, par extension, l'enseignant, à accéder au sens. Comme la compétence phonologique, selon le CECRL, semble être dissociée du texte littéraire, sauf pour la poésie, dans leurs cours de langues les enseignants continuent à travailler peu la lecture à voix haute. C'est justement sur cette dernière et ses subtilités que s'est penchée Marie-Françoise Bourvon, dans son article «Enseigner/apprendre la prosodie par la lecture à voix haute de textes littéraires". Elle revient sur les difficultés des apprenants à lire et à déclamer les textes littéraires, difficultés dues à la fossilisation des compétences en lecture à voix haute acquises en langue maternelle et transférables, par la suite, en langue étrangère. Après s'être interrogée sur les relations qui unissent la ponctuation (pauses de segmentation dans la lecture) à la prosodie, elle met en place deux propositions didactiques à partir de la pièce de théâtre d'Eugène Ionesco, La Cantatrice chauve (mise en scène de Lagarce) et de l'incipit du roman de Marguerite Duras, Le Ravissement de Lol V. Stein, en vue d'une mise en application de l'oralisation des textes littéraires. Dans son article « Enseignement de l'intonation et neurolinguistique", Philippe Martin propose de réfléchir sur l'intonation $d u$ Français Langue Étrangère, qu'il considère comme délaissée dans l'enseignement/apprentissage de l'oral en FLE, et ce, du point de vue neurolinguistique et non pas linguistique. Comme les manuels de FLE se contentent de travailler seulement quelques aspects prosodiques (par le biais des modes déclaratif, impératif, interrogatif), se concentrant plutôt sur la syntaxe et le lexique, il est nécessaire aujourd'hui d'accorder, selon l'auteur, une place importante à l'enseignement du système prosodique du français. En se fondant sur les découvertes récentes en neurolinguistique qui portent sur la synchronisation de la perception des syllabes par les ondes cérébrales, il s'intéresse en particulier aux oscillations delta, thêta et gamma qui permettent de rendre compte de l'élaboration d'une structure prosodique du texte lu (qui peut ou ne peut pas être littéraire; la citation de Genette, supra, est révélatrice à ce propos), de l'eurythmie des groupes accentuels (équilibrage des durées) et de l'alignement de ces groupes avec les unités syntaxiques (par exemple, le cas de la lecture). Claire Pillot-Loiseau et Claudia Schweitzer abordent l'étude de deux figures de style et des figuralismes pour faire de la phonétique en classe de FLE dans leur article: "Allitérations, assonances et figuralismes: de leur histoire à leur utilisation en phonétique du FLE ». Après avoir donné les définitions des assonances (répétition des phonèmes vocaliques), des allitérations (répétition des phonèmes consonantiques), des figuralismes (procédé musical qui consiste à transcrire musicalement une image, un sentiment, une idée) et leur apport à la pédagogie de la prononciation (en compréhension et en production), et présenté aussi bien le rôle du chant dans l'éducation que celui de l'expressivité du discours parlé et chanté, elles insistent sur l'exploitation pédagogique de ces deux figures de style et des figuralismes en prenant pour support des textes littéraires dont certains sont mis en musique.

Le deuxième axe, "Approches phonétiques de la littérature", met en avant les approches pédagogiques qui proposent un traitement efficace de la prononciation. Les approches/méthodes comme, entre autres, l'engagement du corps et de la voix (techniques vocales théâtrales) et la chanson servent de référence afin d'insérer le texte littéraire dans un processus de correction phonétique. Il est question de méthodes, de méthodologies et d'outils utilisables pour l'enseignement de la prononciation à partir de textes littéraires (théâtre, poème, roman), l'objectif principal du numéro étant celui d'accorder une place prépondérante à la mise en application de 
ces méthodes. Ainsi, Corinne Weber propose une réflexion sur les possibilités d'enseignement/apprentissage de la phonétique avec et par le texte littéraire, la didactique de la prononciation devant s'inscrire dans l'oralité (variation et variabilité de la prononciation): "Oralité, littérature et didactique: quelles convergences disciplinaires?». Elle s'intéresse au statut et à la place de l'oralité, ainsi qu'à l'exploitation de la variation (socio-)linguistique dans les corpus littéraires du $\mathrm{xx}^{\mathrm{e}}$ siècle, qui offrent un terrain propice à la didactique de la prononciation du FLE, la composante sociolinguistico-pragmatique étant inextricablement liée à celle-ci. Dans l'optique de trouver des nouvelles approches phonétiques du texte littéraire, Fanny Auzéau invite, dans son article "L'intérêt de l'utilisation du support dramatique : un outil pour entendre et écouter, percevoir et comprendre, parler et s'exprimer en langues étrangères ", à réfléchir sur l'apport de l'utilisation du support dramatique (texte de théâtre) en classe de FLE, support dont le choix peut s'avérer judicieux, et ce à de nombreux titres. Cet outil pédagogique, qui permet une approche participative et active (i.e. monter une pièce de théâtre) met en lumière l'importance du travail de la voix et du corps (prononciation, intonation, position du corps, gestes, etc.). François Blondel vient compléter cette réflexion en proposant certaines techniques d'acteur qui aident à surmonter les appréhensions, à apprendre à mieux articuler (mouvements des organes phonatoires) et à s'approprier les sons, les schémas intonatifs, le rythme de la langue étrangère (du français ici) : «Adopter des techniques d'acteur pour interpréter un texte en classe de langue». La contribution positive des pratiques théâtrales pour améliorer l'oral des étudiants chinois constitue aussi un objet de réflexion pour Christine Cuet : «L'apport des pratiques théâtrales pour l'amélioration de l'oral des étudiants chinois ». L'introduction de l'expression théatrale en classe de FLE se révèle être une pratique dynamique pour travailler l'oral (exercices de communication et d'interaction) et corriger non seulement les erreurs phonétiques et prosodiques mais également transculturelles. L'utilisation des textes littéraires dans les cours de phonétique du FLE est au centre de l'article de Seyed Jamal Mousavishirazi : "Structure sonore d'un texte littéraire ». Il s'intéresse à la beauté de la matière sonore des textes littéraires, de trois genres différents (théâtre: La Cantatrice chauve de Ionesco; roman/autobiographie romancée : La Gloire de mon père de Marcel Pagnol; poésie/ chansons : Le Jardin extraordinaire, les chansons de toute une vie de Charles Trenet), qui se prêtent à l'enseignement/apprentissage de la phonétique du FLE tout en se focalisant sur la nécessité de travailler conjointement la littérature et la phonétique et, plus particulièrement, de se tourner vers l'esthétique des sonorités du texte littéraire qui peut jouer un rôle important dans la motivation des apprenants.

17 Le troisième et dernier axe, "Propositions et applications didactico-pédagogiques ", s'intéresse aux divers aspects pratiques de l'enseignement de la prononciation. Y sont proposées différentes typologies d'activités et d'exercices phonétiques créés à partir des textes littéraires qui, choisis dans un objectif phonétique et/ou prosodique, peuvent aider à améliorer la prononciation des apprenants et à favoriser les interactions et la prise de parole. Pour leur article « Démarches et corrections pour une appropriation des textes littéraires dans leur matérialité phonique et écrite par les apprenants de FLE dès le niveau A1 du CECRL ", Julie Veldeman-Abry et Dominique Abry prennent comme support des exemples littéraires autour desquels sont construites des activités visant à sensibiliser les apprenants, dès le niveau débutant (A1 du CECRL), à la musicalité propre à la langue française de manière à les aider à s'approprier les nouveaux sons, et par extension oralement le texte littéraire, par un travail de 
correction phonétique sur des phénomènes peu développés en classe de langue (le « $\mathrm{e}$ " muet, la diérèse, les enchaînements, etc.). Monique Monville-Burston, Fryni Kakoyianni-Doa et Spyros Armostis insistent, dans leur article « Magie des sons et de la prosodie, et apprentissage de la phonétique par la poésie : "Le Pont Mirabeau" ", sur l'importance de l'enseignement/apprentissage de la phonétique en classe de FLE par l'intermédiaire d'une expérimentation, en cinq étapes et autour d'un poème d'Apollinaire, auprès des étudiants chypriotes hellénophones de niveau B2-C1. Un autre support littéraire pour l'enseignement/apprentissage de la prononciation, celui des comptines à gestes, est proposé par Diane Caussade, Sandra Cornaz et Marina Yezhkova dans leur recherche-action : «Enseignement/apprentissage du FLES aux EFIV : propositions didactiques de l'utilisation de comptines à gestes pour la perception et la production des phonèmes et graphèmes du français ». Elles ont porté leur attention surtout sur l'utilisation des comptines à gestes auprès d'enfants issus de familles itinérantes et de voyageurs (EFIV) pour qui le français n'est qu'une langue seconde (ou étrangère). D'après l'analyse et les résultats obtenus, les autrices concluent que les comptines à gestes sont des supports particulièrement propices à l'enseignement/apprentissage de la perception et de la production des phonèmes du français. René Corona nous offre des « Propositions pour exploiter la poésie en classe de FLE : (re)construire le poème des sons et sa musicalité ». Ressource pédagogique précieuse, la poésie peut faciliter par ses sonorités, sa musicalité et sa matière/matérialité phonique, l'enseignement/apprentissage de la prononciation et de l'intonation, pourvu que les poètes et les poèmes soient judicieusement choisis en fonction des objectifs fixés par l'enseignant et du niveau des apprenants. Dans leur article «Former les enseignants à l'exploitation phonologique d'un texte littéraire ", Maro Patéli et Christos Nikou proposent deux textes de genre littéraire différent: un poème de Paul Verlaine et une nouvelle de Marguerite Yourcenar pour travailler respectivement la compétence réception et la compétence lectorale, travail suivi de conseils/directives/instructions pédagogiques (tel un guide pédagogique) afin de guider les enseignants dans l'exploitation phonologique d'un texte littéraire. Cette section se termine par deux contributions portant sur les nouvelles technologies appliquées à l'enseignement/apprentissage de la prononciation en classe de langue. Avec le développement de l'informatique et de l'ingénierie de la parole, «l'application des nouvelles technologies à l'enseignement des langues ouvre de nouvelles voies à l'expérimentation et à la recherche de méthodes et d'outils qui puissent favoriser l'acquisition des compétences langagières et interculturelles chez les apprenants » (Tomé, $2009: 107)$. Cette situation rejoint notre réflexion car elle allie la langue (du point de vue de sa phonétique), la littérature et ce nouveau domaine d'intérêt: les Nouvelles Technologies de l'Information et de la Communication appliquées à l'enseignement (TICE). Laboratoires de langue multimédias équipés d'outils de pointe, pléthore de ressources Internet, logiciels informatiques conçus pour la correction phonétique (Praat, Winpitch, convertisseur de transcription phonétique, etc.), dispositifs de remédiation destinés aux apprenants du FLE, numérisation des textes littéraires, enregistrements audio des textes littéraires : cette multiplication des Nouvelles Technologies de l'Information et de la Communication a permis de médiatiser et d'améliorer les pratiques enseignantes. Dans son article, « Appropriation multisensorielle du rythme du français : codage rythmique visuel d'extraits filmiques au sein d'un dispositif hybride", Nadia Bacor s'interroge sur l'apprentissage multisensoriel (surtout visuel) $d u$ rythme du français, s'appuyant sur des extraits filmiques (scénario cinématographique) qui en permettent l'appropriation visuelle. Son 
article s'inscrit dans les préoccupations contemporaines de la didactique de la prononciation adossée aux humanités numériques (dispositif hybride ici) et répond à une importante carence en matière de didactique du rythme en FLE dans le contexte des classes de langue. Enfin, l'article de Mario Tomé Díez, « Littérature, phonétique et interactions orales avec les Nouvelles Technologies pour l'apprentissage du Français Langue Étrangère (FLE) ", s'appuie sur le dispositif de formation Campus Virtuel FLE mis en place par l'Université de León. Après avoir fait un état des lieux, l'auteur présente cet outil pédagogique tout en abordant le rôle que les weblogs, les podcasts et les réseaux sociaux jouent dans le développement et l'optimisation de la compétence d'interaction orale ainsi que dans la correction de la prononciation par la littérature.

La problématique de la prononciation étant actuellement au centre des réflexions linguistique et didactique, ce numéro s'est donné pour objectif d'éclairer sous un jour relativement nouveau la place du texte et du discours littéraires dans l'enseignement/ apprentissage de la phonétique du Français Langue Étrangère et/ou seconde. Il vise, avant tout, à encourager les enseignants à travailler conjointement la phonétique et la littérature de façon efficace et organisée en leur apportant des connaissances, des outils et des techniques de terrain, et à leur faire prendre conscience qu'en associant la culture à la phonétique dans un processus d'enseignement (et/ou de recherche) on peut optimiser l'apprentissage d'une langue étrangère, en l'occurrence du français.

Nous tenons à exprimer nos remerciements les plus sincères aux contributeurs du numéro, ainsi qu'aux membres du comité de lecture qui ont accompli dans les délais prévus un travail sérieux et appliqué.

\section{BIBLIOGRAPHIE}

BARTHES R., 1963, « Le message publicitaire, rêve et poésie », Communication \& Langages, $\mathrm{n}^{\circ}$ 7,

p. 91-96. Disponible sur :

<https://www.persee.fr/doc/colan_1268-7251_1963_num_7_1_4840>.

CONSEIL DE L'EUROPE / Division des Politiques Linguistiques (Strasbourg), 2001, Un Cadre européen

commun de référence pour les langues. Apprendre, Enseigner, Évaluer

(CECRL), trad. par S. Lieutaud, Paris, Didier.

Disponible sur : <https://rm.coe.int/16802fc3a8>.

DUFEU B., 2008, «L'importance de la prononciation dans l'apprentissage d'une langue étrangère », Franc-parler.org. Disponible sur :

$<$ http://www.francparler-oif.org/images/stories/dossiers/phonetique_dufeu.htm>.

GENETTE G., 1966, Figures I, Paris, Éditions du Seuil, coll. « Tel Quel ».

GUIMBRETIÈRE É., 1994, Phonétique et enseignement de l'oral, Paris, Didier Hatier.

LAURET B., 2007, Enseigner la prononciation du français : questions et outils, Paris, Hachette, coll. «f $»$. 
LE CLÉZIO J.-M. G., 2008, « Dans la forêt des paradoxes », Discours de réception du prix Nobel de littérature prononcé le dimanche 7 décembre 2008 à Stockholm. Disponible sur :

<http://www.nobelprize.org/nobel_prizes/literature/laureates/2008/clezio-lecture_fr.html>.

SAUVAGE J. et BILLIÈRES M., 2019, « Enseigner la phonétique d'une langue étrangère : Bilan et

perspectives ", Cahiers de l'Acedle, vol. 16, $\mathrm{n}^{\circ} 1$. Disponible sur :

$<$ https://journals.openedition.org/rdlc/4234>.

TOMÉ M., 2009, « Compétences orales et outils de communication Web dans un projet de

télécollaboration pour l'apprentissage du français langue étrangère », Revue de l'éducation à

distance, vol. 23, $\mathrm{n}^{\circ}$ 1, p. 107-126. Disponible sur :

<http://www.jofde.ca/index.php/jde/article/view/578>.

ÜBERSFELD A., 1997, «Éditorial d'introduction thématique au numéro », AS/SA, nº3, p. 114-118.

Disponible sur :

$<$ http://www.chass.utoronto.ca/french/as-sa/ASSA-No3/AU1.html>.

VALÉRY P., 2002 [1944], « Leçon inaugurale du cours de poétique du Collège de France », dans

Variété III, IV et V, Paris, Gallimard, coll. « Folio essais ».

\section{NOTES}

1. Le marché éditorial du FLE est relativement riche en manuels conçus pour la phonétique et les approches sont des plus variées : gestuelle, paysagiste, théâtrale, musicale... Outils indispensables pour la formation des enseignants, certes, ces manuels au côté trop spécialisé, voire hermétique, sont peu et rarement utilisés en classe car inaccessibles aux apprenants étrangers (parfois même aux enseignants). Dans la plupart des méthodes de FLE, la phonétique est rejetée en fin de leçon : les activités y sont courtes et peu explicites, et il appartient, le plus souvent, à l'enseignant et/ou à l'apprenant d'aller chercher des activités appropriées.

2. «Les exercices de prononciation proposés sont en général fort succincts et ne permettent pas d'espérer une maîtrise du problème considéré. On trouve souvent dans le cadre d'une leçon un exercice sur un phénomène de prononciation, par exemple le rythme de la langue française, puis on passe dans l'unité suivante à un autre thème, laissant supposer que le problème abordé précédemment est résolu. On a l'impression d'un assemblage d'exercices juxtaposés dont la progression est souvent absente ou parfois contre-productive. [...] La prononciation n'est pas intégrée dans l'ensemble du processus d'apprentissage. Elle apparait comme un exercice isolé, comme une obligation à remplir pour répondre aux critères didactiques du moment » (Dufeu, 2008).

3. « S'ils [phonèmes] ne sont pas acquis ou appris, cela entraînera un déficit d'information et des malentendus peuvent surgir. Quels sont les risques de fréquence de ces malentendus et leur gravité ? Quelle priorité doit-on leur donner? Ici, la question de l'âge ou de la période de l'apprentissage pendant laquelle il est préférable d'apprendre ces différences se complique du fait que la fossilisation des erreurs est très importante au niveau phonétique. Rendre conscient des erreurs phonétiques et désapprendre des comportements devenus automatiques peut être beaucoup plus coûteux (en temps et en efforts) lorsque l'apprenant s'est approprié une forme approximative par rapport à la norme que cela l'aurait été au début de l'apprentissage, notamment précoce » (Conseil de l'Europe, $2001: 105$ ).

4. La poésie ne prend toute sa valeur qu'au moment de sa diction : "c'est l'exécution du poème qui est le poème. En dehors d'elle, ce sont des fabrications inexplicables, que ces suites de paroles, curieusement assemblées ", affirmait Paul Valéry (2002: 838). 
5. «S'y ajoute ce qu'on pourrait appeler une poétique phonique, en relation avec les signes auditifs : les phonèmes d'abord, et la question se pose de leur sens et de leur effet, ensuite les lexèmes, leurs récurrences et leurs parallélismes; le rythme enfin qui engage la question des divers modes d'organisation sonore du verbal et de la versification. Il est intéressant de voir ces trois éléments se conjuguer par exemple pour expliquer l'effet des rimes et des assonances » (Übersfeld, 1997 : 117).

6. Réciter un poème ou jouer une scène de théâtre peut contribuer à la gestion de l'anxiété linguistique de certains apprenants.

7. Rappelons ce que Roland Barthes (1963 : 95) note à propos du message publicitaire (slogan) : «les critères du langage publicitaire sont ceux-là même de la poésie: figures rhétoriques, métaphores, jeux de mots [...] tout message publicitaire dit et raconte ».

8. Des revues et ouvrages spécialisés préconisent l'exploitation des textes littéraires dans l'apprentissage du Français Langue Étrangère (FLE). À titre indicatif: Michel Benamou, 1979, Pour une Nouvelle Pédagogie du Texte Littéraire, Paris, Librairies Hachette et Larousse ; Isabelle Taillandier et Caroline Bouchery, 2009, Le FLE par les textes : Littérature et activités de langue, Paris : Belin, coll. «FLE »; Fridériki Tabaki-Iona, Argyro Proscolli et Kyriakos Forakis (dir.), 2010, La place de la littérature dans l'enseignement du FLE. Actes du Colloque international des 4 et 5 juin 2009, Athènes, Presses de l'Université d'Athènes; Marjolaine Pierré et Frédérique Treffandier, 2012, Jeux de théâtre, Grenoble, PUG, coll. «Les outils malins du FLE »; Mariana Arzate-Otamendi et Adelina Velasquez-Herrera, 2012, «Intégration du texte littéraire dans la classe de langue: une expérience pédagogique mise en œuvre en contexte universitaire", Synergies Mexique, no 2, p. 41-55; Yening Bao, 2012, «De la dimension poétique des textes littéraires en classe de FLE », Synergies Chine, no 7, p. 169-178; Martine Fiévet, 2013. Littérature en classe de FLE, Paris, CLE International, coll. « Techniques et pratiques de classe »; Jean-Marc Defays, Olivier Delbard et Sarra Hammami, 2014. La Littérature en classe de FLE : états de lieux et nouvelles perspectives, Paris, Hachette FLE, coll. «F »; Anne Godard, 2015, La littérature dans l'enseignement du FLE, Paris, Didier, coll. «Langues \& didactique». Certes, ces publications traduisent un regain d'intérêt pour l'introduction du texte littéraire en classe de FLE, cependant, elles abordent peu les modalités de son exploitation à traves le prisme de la phonétique.

9. On pourrait établir une typologie des textes littéraires en fonction de leur degré d'oralité et, pour être plus précis, en fonction de leur organisation phonématique pour créer l'illusion de la parole. Le discours du roman, de Rabelais à Queneau, en passant par Flaubert, Stendhal, Zola, Proust, en constitue l'exemple le plus frappant.

10. Voir par exemple les rimes dans Cyrano de Bergerac de Rostand ou dans le théâtre racinien, les phrases élégantes dans À la recherche du temps perdu de Proust, les vers de Rimbaud ou de Victor Hugo, les jeux de mots et le but moralisateur des fables (Ésope, Jean de La Fontaine...), les paroles de Charles Trenet ( La mer») ou encore les paroles de Jacques Plante et la musique de Charles Aznavour dans la chanson de ce dernier «Les comédiens», etc. On pourrait également citer : « pour qui sont ces serpents qui sifflent sur vos têtes » (Racine, Andromaque, V, 5) pour le travail de « $\mathrm{s}$ »; « je le vis, je rougis, je pâlis à sa vue » (Racine, Phèdre, I, 3), pour le travail du rythme; le poème de Prévert «Les oiseaux du souci», pour le travail du « $u$ » et des semivoyelles : «Plumes de pluie pluie de plumes / depuis que tu n'es plus je ne sais plus / je ne sais plus où j'en suis... suaire de pluie pluie de suie..."; le travail des groupes consonantiques, difficiles pour plus d'un apprenant étranger : «De truands transistors trituraient les trottoirs... » (Raymond Queneau); l' élaboration musicale de la phrase chez Flaubert (rythme oral du "gueuloir», chasse aux répétitions et aux assonances). Flaubert s'interrompait régulièrement pour gueuler (d'où le nom de gueuloir) ses textes, les mettant à l'épreuve de l'oral (de la lecture à voix haute) pour vérifier la cohérence et la pureté de chaque proposition : "Une phrase est viable, disait-il, quand elle correspond à toutes les nécessités de la respiration. Je sais qu'elle est bonne lorsqu'elle peut être lue tout haut. Les phrases mal écrites, écrivait-il dans la préface des 
Dernières Chansons de Louis Bouilhet, ne résistent pas à cette épreuve ; elles oppressent la poitrine, gênent les battements du cœur et se trouvent ainsi en dehors des conditions de la vie.", Guy de Maupassant, "Gustave Flaubert », étude préfaçant le livre Lettres à George Sand, par Gustave Flaubert, 1884 ;Baudelaire et ses « Correspondances »...

11. Jérémi Sauvage et Michel Billières (2019), responsables d'un numéro des Cahiers de l'Acedle, intitulé « Enseigner la phonétique d'une langue étrangère : Bilan et perspectives », réfléchissent aux raisons tant historiques qu'épistémologiques «qui ont conduit à [...] une crise dans l'enseignement de la composante phonético-phonologique des langues étrangères ", ainsi qu'à la place « restreinte qu'occupe aujourd'hui la phonétique corrective dans la didactique des langues étrangères ».

\section{AUTEURS}

\section{FLORENTINA FREDET}

Université Sorbonne Nouvelle-Paris 3

\section{CHRISTOS NIKOU}

Sorbonne Université- Faculté des Lettres, CRLC/EA 4510 